\title{
MALAISE aux jardins : des milliers d'abeilles perdues pour la science? \\ Monographie et typologie de collectes d'abeilles mortes dans les serres de jardins potagers à Rennes (France)
}

par Patrick JÉZÉQUEL*

Publication : juillet 2020

\begin{abstract}
Résumé. Les serres de jardins potagers capturent de manière passive les Abeilles solitaires. Les résultats d'une saison de collecte montrent la valeur inexploitée de ce nouveau moyen d'inventaire pour les réseaux amateurs et universitaires.
\end{abstract}

Abstract. Greenhouses of allotments and kitchen gardens collect wild bees at a magnitude which might be seen as a passive sampling method to monitor bees at local or national level.

Mots-clés. Tentes MALAISE ad hoc, méthode passive, inventaires, réseau national de collecte.

Keywords. Improvised MALAISE traps, passive capture, inventory, national collecting network.

\section{Introduction}

En mars 2017, je rendais visite à un ami maraîcher lorsqu'en visitant sa nouvelle serre, j'aperçus Andrena fulva gisant sur un pli de la bâche transparente dans un angle de la serre, prisonnière, morte depuis quelques jours. Cette abeille est identifiable à vue, je ne l'avais donc jamais capturée. Je pus ainsi compléter ma collection de référence. L'identification à l'espèce de la majorité des Abeilles nécessite une capture létale pour un travail sous binoculaire. Alors que les naturalistes qui refusent le piégeage sont nombreux, cette nouvelle méthode de collecte fortuite aurait l'avantage d'être passive et comparable aux tentes MALAISE. Mais avec quels résultats et quels biais?

C'est ainsi que commença une campagne de collecte régulière dans la serre Perma $G$ (figure 1). Des prospections au mois d'août dans d'autres jardins de l'agglomération rennaise ont permis d'esquisser une typologie des différentes serres utilisées par les jardiniers, ainsi qu'une illustration des inventaires qu'offre cette méthode de collecte.

* Bretagne Vivante, 19 rue de Gouesnou, BP 62132, F-29221 Brest cedex 2 - Observatoire des Abeilles, 68 rue du Onze Novembre, F-59148 Flines-lez-Raches - gomjabar35@hotmail.fr

\section{Matériel et Méthode}

Protocole et modalités de capture

Les abeilles capturées dans la serre Perma $G$ ont été collectées toutes les deux semaines au cours des mois de mars, avril, mai et août, et une fois par semaine en juin et juillet, mois correspondant au pic d'activité de ces insectes dans le département de l'Ille-et-Vilaine. Prisonnières de la serre, les Abeilles meurent d'épuisement et s'amassent dans les plis de la bâche aux angles sud-est et sud-ouest (figures 1-2) exposés au soleil. Il est possible de ne pas collecter les spécimens durant un mois ou plus, mais la chaleur, le rayonnement solaire et l'humidité présente dans la serre peuvent détériorer les individus (décoloration, moisissure, décomposition, bris). Pour les autres serres de la région rennaise, la collecte a été réalisée au mois d'août en une seule fois. Les insectes sont récupérés au moyen d'un récipient dans lequel on fait glisser les individus prisonniers des plis de la bâche (figure 2). Une pince souple peut être utilisée pour récupérer les individus peu accessibles - par exemple dans les petits espaces entre les arceaux - ou en début et fin de saison lorsque leur nombre est réduit, permettant un premier tri entre Abeilles et autres Insectes. 


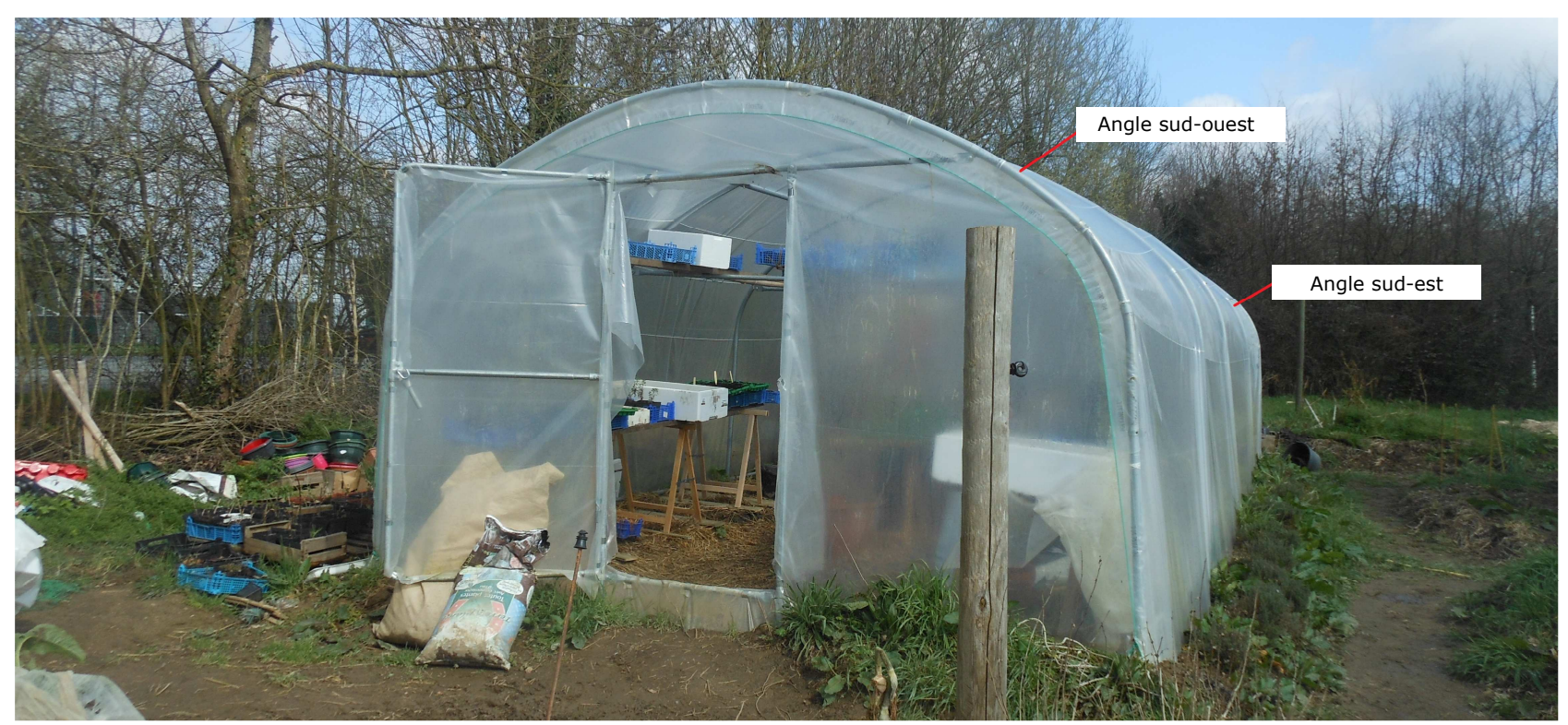

Figure 1. Serre Perma G, Rennes.

Photo : P. JÉzÉQUEL mars 2018

Très peu de " casse " est observée lors de ces manipulations; il est rare par exemple que les deux antennes ou les deux mêmes pattes d'un individu soient brisées, et très rare que la tête se soit détachée, sauf s'il y a eu de la condensation.

Un premier tri est effectué sur place; les petits insectes sont regroupés et triés plus tard sous loupe binoculaire. Le produit des collectes est comparable à celui d'une tente MALAISE avec un gros pourcentage de Diptères, des Papillons et quelques Coléoptères. Les collectes sont placées au moins 48 heures au congélateur avant d'être exploitées. La mise en collection des Abeilles s'effectue après réhydratation, en les immergeant complètement dans l'eau pendant une heure ou plus. Toutes les serres étudiées sont situées dans des jardins potagers associant des cultures de légumes et de plantes vivaces de tous types, ainsi que quelques arbres fruitiers. Certaines parcelles sont plus ou moins entretenues.

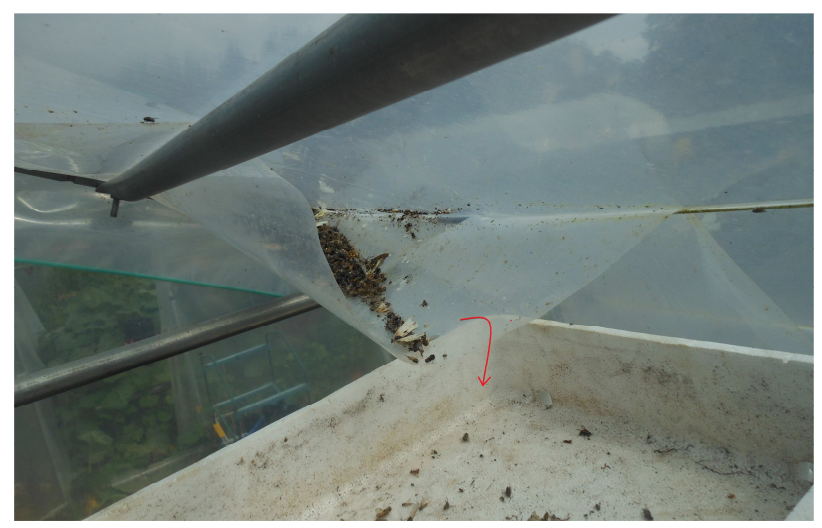

Figure 2. Collecte des abeilles (Serre Perma $G$ ). Photo : P. JÉZÉQUEL juin 2018
Ainsi, il subsiste parfois des ronces et herbes hautes en bordure. Il arrive également que des parcelles restent en friche en attendant un nouveau locataire. Leur localisation est variable, même si elle se concentre essentiellement dans le secteur périurbain. Les serres étudiées sont utilisées pour la culture de tomates.

\section{$\underline{\text { Recherche et prospection des parcelles }}$}

Afin d'optimiser le temps de terrain, une première recherche peut être faite par image satellite pour localiser les jardins et la présence de serres. Il existe beaucoup de petits potagers de 10 ou 15 parcelles qui ne sont pas répertoriés dans les réseaux officiels des mairies ou des associations. L'idéal est de prospecter le week-end lorsque les jardiniers sont là, parfois aussi le soir vers 18 ou 19 heures à partir de mai. Bien entendu, il est nécessaire de demander l'autorisation, de parler avec les voisins et de se faire connaitre. L'intérêt général pour les Abeilles facilite le contact et il n'est pas rare de discuter une demi-heure sur le sujet en montrant la diversité des spécimens collectées.

\section{Typologie des serres}

Toutes les serres capturent, certaines plus que d'autres, mais peu collectent. On dit d'une serre qu'elle " collecte " lorsque les abeilles piégées peuvent être récupérées car elles ne sont pas tombées au sol. Vers la fin juillet 2017, une campagne de prospection a été menée dans les jardins partagés et « ouvriers » de Rennes et de son 
agglomération. Des centaines de parcelles ont été visitées mais moins de $50 \%$ d'entre elles possédaient une serre.

Lors de cette campagne, nous avons identifié 3 types de serre :

- Les serres dites « légères" (figure 3) : elles sont généralement vendues en jardinerie et portent leur nom du fait de leur armatures légères. La toile plastique est cousue en usine et s'ajuste aux armatures.

- Les serres à " bâche recouvrante " (figures 1 et 4) : elles sont caractérisées par une bâche tendue lors du montage et enterrée dans le sol.

- Les serres dites " artisanales " (figures 5-7): elles sont bricolées directement par les jardiniers. Elles peuvent être de toutes sortes : intégralement ou partiellement bâchées, avec ou sans toit en PVC, armature métallique ou en bois.

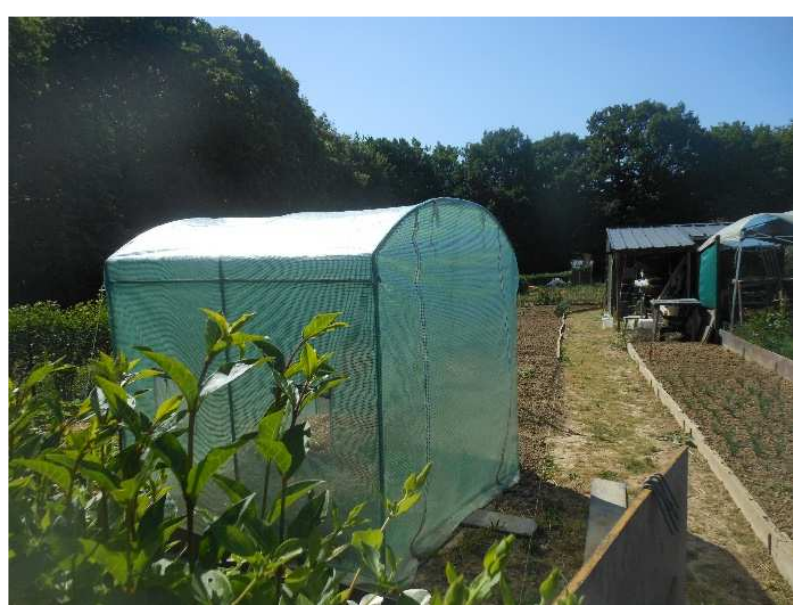

Figure 3. Serre « grand public » vendue en jardinerie. Photo : P. JÉZÉQUEL 2017

Concernant leurs capacités de collecte, les serres dites légères sont les moins performantes (figure 3). Non pas qu'elles ne retiennent pas prisonnières les Abeilles comme les autres serres, mais, à cause de l'absence totale ou partielle de replis dans leurs armatures, les individus tombent au sol en mourant et s'y décomposent. Ce phénomène est accentué par l'armature flexible de la serre, les vibrations engendrées par le vent faisant tomber les quelques Abeilles accrochées aux coutures. Nous avons tout de même pu trouver quelques rares spécimens de petites espèces accrochées aux coutures telles que Lasioglossum morio et L. malachurum.

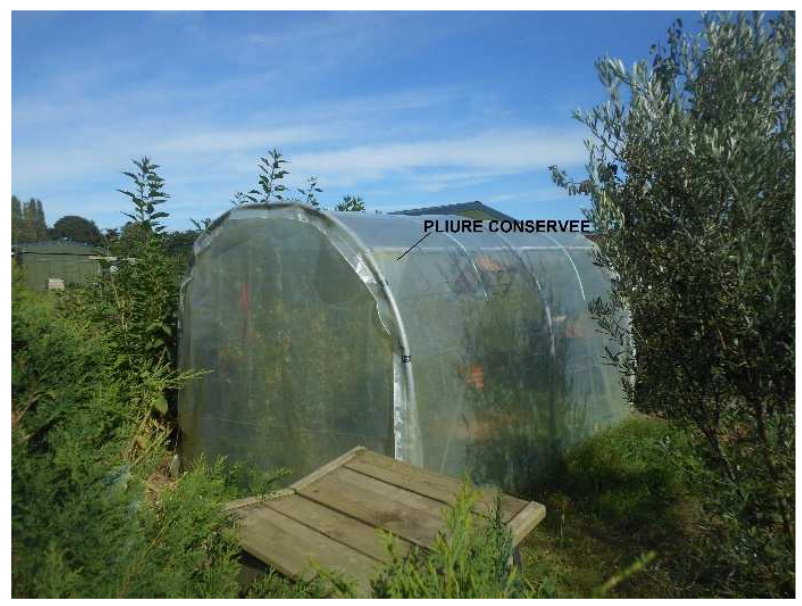

Figure 4. Serre Gayeulles-est numéro 1 à Rennes Photo : P. JÉzÉQUEL 2017

Les serres à " bâche recouvrante » sont nettement plus intéressantes. Sous réserve que le jardinier n'ait pas coupé les plis qui se forment aux angles (figure 4), elles peuvent fournir un grand nombre de spécimens facilement récoltables. En effet, ces plis forment des réceptacles pour les Abeilles épuisées qui s'y laissent mourir. Les Abeilles peuvent aussi être collectées le long des armatures épaisses sur le petit plat, coincées contre le plastique. Toutefois, la taille d'un même type de serres peut affecter la quantité potentielle d'Abeilles collectées. Les plus petites à une ouverture piègent moins que les grandes à deux ouvertures (figure 4).

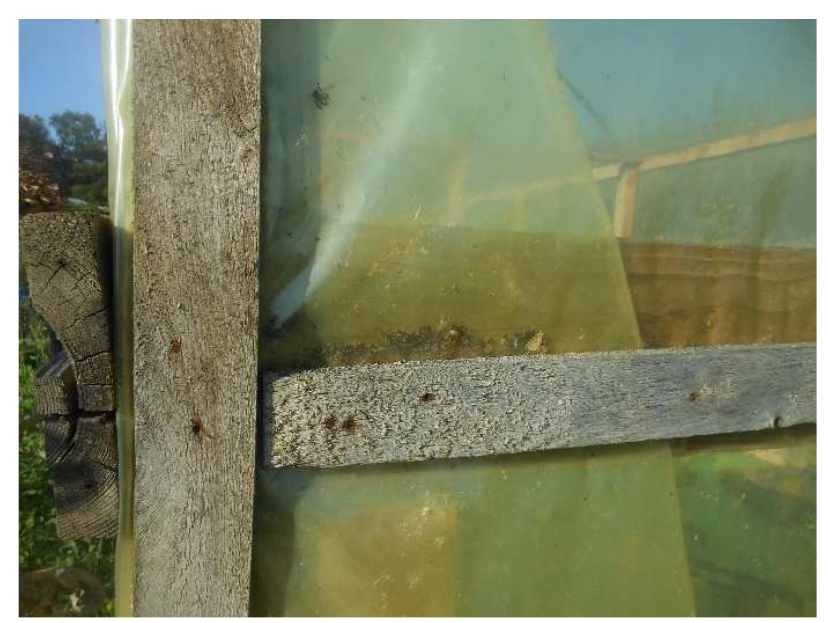

Figure 5. Serre Gayeulles-est numéro 3 à Rennes Photo : P. JÉZÉQUEL 2017

Les serres " artisanales " ont un potentiel de collecte très variable. Celles dont le toit est en tôle de plastique ondulée transparente laissent échapper les Abeilles au niveau des bordures, 


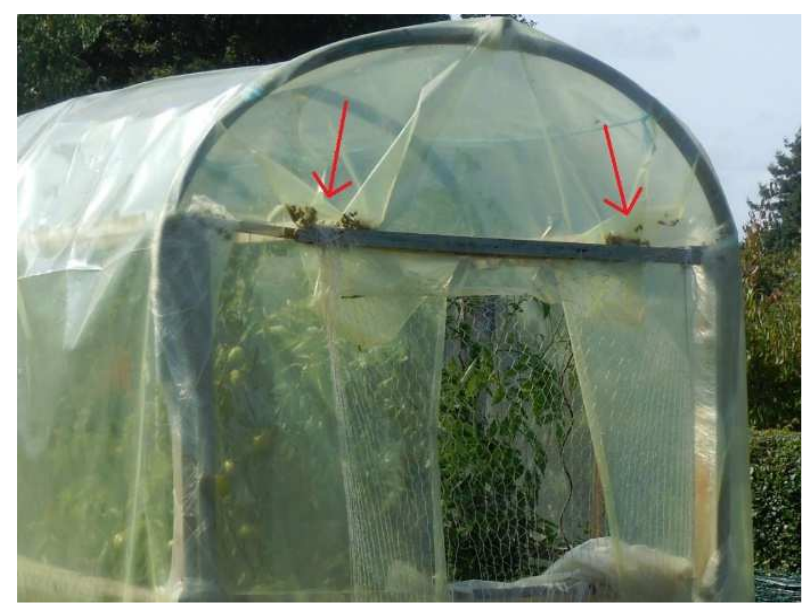

Figure 6. Serre Taupinais numéro 83 à Rennes Photo : P. JÉzÉQUEL 2017

entre le creux de l'ondulation et l'élément qui soutient la tôle. Une serre artisanale qui capture et collecte est généralement constituée d'une armature en bois et recouverte d'une bâche plastique tenue soit par des petites lattes en bois vissées ou pointées (figures 5-6), soit par une ficelle (figure 7). Dans l'exemple de la figure 5, on voit qu'il est difficile d'accéder aux Abeilles sans une pince souple, et l'extraction entraîne parfois la casse des Insectes. Si les lattes de bois sont vissées, il est très facile de les dévisser en partie, de placer un récipient et de collecter puis revisser. Si les lattes sont pointées, il faut prévoir des vis et revisser pour l'année suivante.

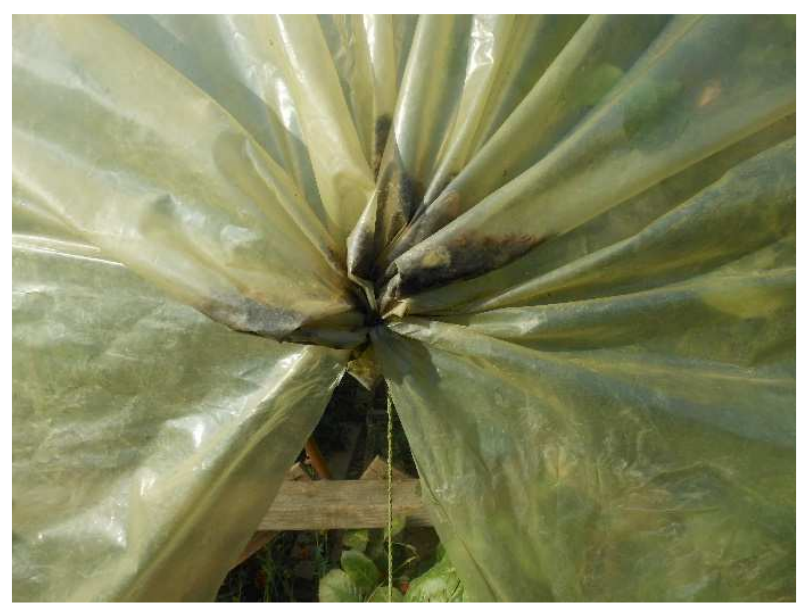

Figure 7. Serre Gayeulles-est numéro 2 Photo : P. JÉZÉQUEL 2017

\section{Un cas particulier}

En septembre 2017, une visite dans un jardin de Saint-Aubin a permis de confirmer que les serres légères capturaient mais ne collectaient pas. Dès l'entrée dans le jardin, il apparut comme évident qu'aucune des serres ne serait intéressante car elles étaient toutes du modèle léger disponible en jardinerie (figure 3). Pourtant, la propriétaire témoigna qu'elle venait de nettoyer sa serre et qu'elle avait jeté une pleine boîte d'insectes. En réalité, sa serre est utilisée à la fois comme abri et comme lieu de travail et de semis : elle y passe du temps et elle y a donc installé un drap comme

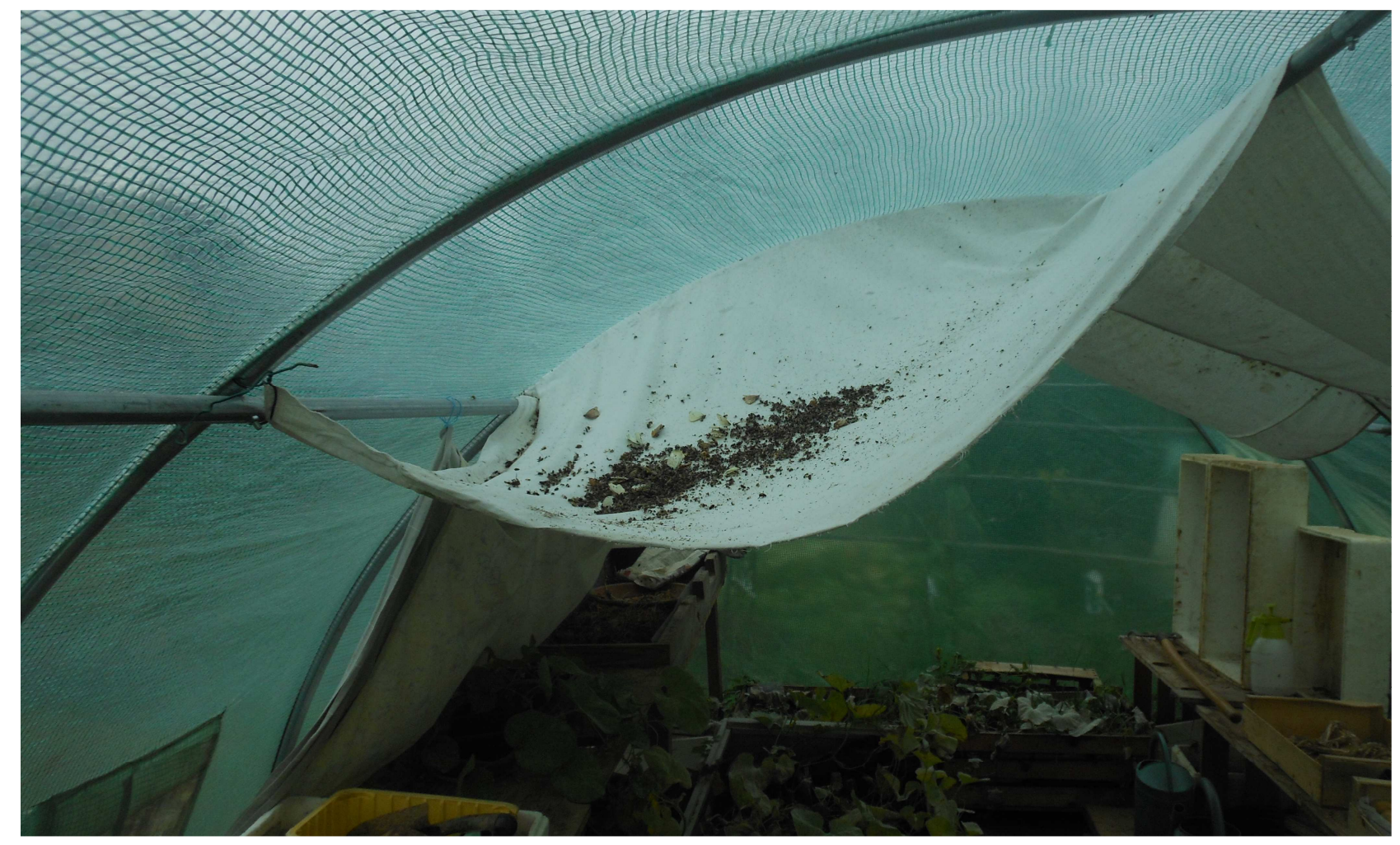

Figure 8. Serre numéro 1 à Saint-Aubin-du-Cormier Photo : P. JÉZÉQUEL 2018 
" faux plafond " pour faire de l'ombre. Le résultat est que cette serre collecte comme le montre la photo prise en août 2018 (figure 8 ; la toile a été secouée et détendue, les Abeilles étant plus uniformément réparties à l'origine). Il est donc possible de collecter dans une serre de ce type ; il suffit d'aménager un support pour que les Abeilles ne tombent pas au sol.

\section{Résultats et discussion}

Jardin de la Taupinais Perma $G$ à Rennes : monographie d'une saison de collecte (2017)

Ce jardin se situe en périphérie de Rennes, dans une zone semi-agricole non-intensive, constituée de prairies de fauches, de zones humides, de boisements et de quelques habitations. La parcelle de $1500 \mathrm{~m}^{2}$ est entourée de haies bocagères stratifiées composées de Noisetiers, Chênes, Aubépines et Ronces. La serre étudiée a été mise en place en 2017 en vue d'une activité professionnelle de maraîchage. Elle se situe en bordure de parcelle, parallèlement au linéaire bocager, avec de grandes portes de chaque côté (figure 1).

423 Abeilles appartenant à 70 espèces (AMIET 1996 ; AMIET et al. 1999-2017 ; PATINY 2010 ; PAULY 2015, 2016) ont été collectées en 2017 dans la serre Perma G (tableaux I-II). Les Halictidae représentent $61,4 \%$ du total avec 260 individus dont 173 Lasioglossum et 14 Sphecodes, les Andrenidae 22,7 \% avec 96 individus dont 48 Micrandrena. Les Apidae composent 9,5\% de l'inventaire, avec 40 individus collectés, dont 21 Nomada (13 N. flavoguttata).

Tableau I. Collectes du jardin de la Taupinais (Rennes) : bilan.

\begin{tabular}{lcc} 
Famille & Nombre & Proportion \\
\hline Halictidae & 260 & $61,46 \%$ \\
Andrenidae & 96 & $22,70 \%$ \\
Apidae & 40 & $9,45 \%$ \\
Megachilidae & 19 & $4,50 \%$ \\
Colletidae & 5 & $1,18 \%$ \\
Mellitidae & 3 & $0,7 \%$ \\
Total & $\mathbf{4 2 3}$ & $100 \%$
\end{tabular}

Dix-neuf individus (soit $4 \%$ du total des spécimens récoltés) appartiennent à la famille des Megachilidae. Enfin, la famille des Colletidae est représentée par 5 Hylaeus et celle des Melittidae par 3 individus appartenant à l'espèce Melitta leporina. L'ensemble des données brutes est disponible dans le tableau II.

Les dates correspondent à la collecte du premier individu de l'espèce. Pour des raisons de temps, les Micrandrena n'ont pas toutes été déterminées ou validées. Les femelles du groupe simplex sont inséparables et non comptées (NC) comme espèces. Apis mellifera n'a pas été collectée ni comptée ; on peut estimer qu'il y a eu entre 50 et 100 Abeilles domestiques capturées, des ruchers se situant à 50 mètres de la serre. Il reste enfin à confirmer l'identification de 18 Lasioglossum (spp.).

Voici la liste des espèces avec une mise en valeur des nouvelles espèces pour la région Bretagne ${ }^{* *}$ et pour le département d'Ille-et-Vilaine (35)* (APOIDEA ARMORICANA, 2018) collectées en 2017 :

Andrenidae (15 espèces) : Andrena angustior, $A$. bicolor, A. carantonica, A. dorsata, A. flavipes, $A$. florea, A. fulvago, A. humilis, A. minitula, $A$. minituloides, A. trimenara, A. ovatula, A. wilkella. Panurgus calcaratus, Panurgus dentipes.

Apidae (15) : Apis melifera, Bombus lucorum, B. pascuorum, B. pratorum, B. terrestris, Ceratina cucurbitina, C. cyanea, Eucera nigrescens, Nomada bifasciata, N. flavoguttata, N. fucata, N. marshamela, $N$. ruficornis, $N$. succincta, $N$. zonata.

Colletidae (4) : Hyleus communis, $H$. dilatatus, $H$. hyalinatus, $H$. variegatus.

Halictidae (23) : Halictus langobardicus, $H$. maculatus, $H$. scabiosae, $H$. subauratus, $H$. tumulorum, Lasioglossum calceatum, $L$. lativentre, L. laticeps, L. leucozonium, $L$. malachurum, L. minutissimum, L. morio, $L$. pauxilum, L. sexnotatum, $L$. villosolum, $L$. xanthopus, L. zonulum, Sphecodes ephipius, S. gibus, S. hyalinatus, $S$. monilicornis, $S$. puncticeps, S. reticulatus*.

Megachilidae (12) : Anthidium manicatum, Chelostoma distinctum**, Heriades truncorum, Hoplitis leucomelana**, Hoplitis tridentata**, Megachile centuncularis, M. lagopoda, Osmia caerulescens, $O$. cornuta, $O$. gallarum**, $O$. bicornis, Stelis punctulatissima.

Melittidae (1) : Melitta leporina. 
Tableau II. Collectes du jardin de la Taupinais (Rennes) : détail.

Coordonnées Lambert $93:$ X $348831-$ Y 6788055. $\mathrm{F}=$ femelle. $\mathrm{M}=$ mâle.

\begin{tabular}{|c|c|c|c|c|c|c|}
\hline Espèce & Effectif & Date & Récolteur & Déterminateur 1 & Déterminateur 2 & 2 Collection \\
\hline \multicolumn{7}{|c|}{ Récoltes 2017} \\
\hline \multicolumn{7}{|l|}{ Andrenidae } \\
\hline Andrena angustior & $4 \mathrm{~F}$ & 08/04/2017 & P. JÉZÉQUEL & P. JÉZÉQUEL & D. GENoud & P. JÉZÉQUEL \\
\hline Andrena bicolor & $2 \mathrm{~F}$ & $01 / 05 / 2017$ & P. JÉZÉQUEL & P. JÉZÉQUEL & D. GENOUD & P. JÉZÉQUEL \\
\hline Andrena dorsata & $5 \mathrm{~F}$ & 01/07/2017 & P. JÉZÉQUEL & P. JÉZÉQUEL & D. GENOUD & P. JÉZÉQUEL \\
\hline Andrena flavipes & $1 \mathrm{~F}+1 \mathrm{M}$ & 01/07/2017 & P. JÉZÉQUEL & P. JÉZÉQUEL & D. GENOUD & P. JÉZÉQUEL \\
\hline Andrena florea & $1 \mathrm{~F}$ & $01 / 06 / 2017$ & P. JÉZÉQUEL & P. JÉZÉQUEL & D. GENOUD & P. JÉZÉQUEL \\
\hline Andrena fulva & $1 \mathrm{~F}$ & $17 / 03 / 2017$ & P. JÉZÉQUEL & P. JÉZÉQUEL & D. GENOUD & P. JÉZÉQUEL \\
\hline Andrena fulvago & $6 \mathrm{~F}+1 \mathrm{M}$ & $14 / 05 / 2017$ & P. JÉZÉQUEL & P. JÉZÉQUEL & D. GENOUD & P. JÉZÉQUEL \\
\hline Andrena humilis & $2 \mathrm{M}$ & $14 / 05 / 2017$ & P. JÉZÉQUEL & P. JÉZÉQUEL & D. GENOUD & P. JÉZÉQUEL \\
\hline Andrena minitula & $4 \mathrm{~F}+1 \mathrm{M}$ & $01 / 05 / 2017$ & P. JÉZÉQUEL & P. JÉZÉQUEL & D. GENOUD & P. JÉZÉQUEL \\
\hline Andrena minituloides & $1 \mathrm{~F}$ & $14 / 05 / 2017$ & P. JÉZÉQUEL & P. JÉZÉQUEL & D. GENOUD & P. JÉZÉQUEL \\
\hline Andrena (Micrandrena) spp. & $39 \mathrm{~F}+3 \mathrm{M}$ & 05 à $07 / 2017$ & P. JÉZÉQUEL & P. JÉZÉQUEL & & P. JÉZÉQUEL \\
\hline Andrena ovatula & $3 \mathrm{~F}$ & $01 / 07 / 2017$ & P. JÉZÉQUEL & D. GENOUD & & P. JÉZÉQUEL \\
\hline Andrena trimmenara & $1 \mathrm{~F}$ & $01 / 07 / 2017$ & P. JÉZÉQUEL & D. GENOUD & & P. JÉZÉQUEL \\
\hline Andrena wilkella & $3 \mathrm{~F}+1 \mathrm{M}$ & $01 / 06 / 2017$ & P. JÉZÉQUEL & P. JÉZÉQUEL & D. GENOUd & P. JÉZÉQUEL \\
\hline Panurgus calcaratus & $2 \mathrm{~F}$ & $01 / 08 / 2017$ & P. JÉZÉQUEL & P. JÉZÉQUEL & D. GENOUD & P. JÉZÉQUEL \\
\hline Panurgus dentipes & $1 \mathrm{M}$ & 01/07/2017 & P. JÉZÉQUEL & P. JÉZÉQUEL & D. GENOUD & P. JÉZÉQUEL \\
\hline \multicolumn{7}{|l|}{ Apidae } \\
\hline Apis mellifera & $\mathrm{NC}$ & $17 / 03 / 2017$ & P. JÉZÉQUEL & P. JÉZÉQUEL & & P. JÉZÉQUEL \\
\hline Bombus lucorum & $1 \mathrm{~F}$ & $21 / 05 / 2017$ & P. JÉZÉQUEL & G. MAHÉ & & P. JÉZÉQUEL \\
\hline Bombus pascuorum & $6 \mathrm{~F}$ & $01 / 07 / 2017$ & P. JÉZÉQUEL & G. MAHÉ & & P. JÉZÉQUEL \\
\hline Bombus pratorum & $1 \mathrm{~F}$ & $21 / 05 / 2017$ & P. JÉZÉQUEL & G. MAHÉ & & P. JÉZÉQUEL \\
\hline Bombus terrestris & $1 \mathrm{~F}$ & 08/04/2017 & P. JÉZÉQUEL & P. JÉZÉQUEL & G. MAHÉ & P. JÉZÉQUEL \\
\hline Ceratina cyanea & $2 \mathrm{~F}+4 \mathrm{M}$ & 01/07/2017 & P. JÉZÉQUEL & P. JÉZÉQUEL & & P. JÉZÉQUEL \\
\hline Ceratina cucurbitina & $1 \mathrm{~F}$ & $01 / 08 / 2017$ & P. JÉZÉQUEL & P. JÉZÉQUEL & & P. JÉZÉQUEL \\
\hline Eucera nigrescens & $2 \mathrm{~F}+1 \mathrm{M}$ & 02/06/2017 & P. JÉZÉQUEL & P. JÉZÉQUEL & & P. JÉZÉQUEL \\
\hline Nomada bifasciata & $1 \mathrm{~F}$ & $17 / 04 / 2017$ & P. JÉZÉQUEL & P. JÉZÉQUEL & É. DUFRÊNE & P. JÉZÉQUEL \\
\hline Nomada flavoguttata & $10 \mathrm{~F}+3 \mathrm{M}$ & 08/04/2017 & P. JÉZÉQUEL & P. JÉZÉQUEL & É. DUFRÊNE & P. JÉZÉQUEL \\
\hline Nomada fucata & $2 \mathrm{~F}$ & $01 / 07 / 2017$ & P. JÉZÉQUEL & P. JÉZÉQUEL & É. DUFRÊNE & P. JÉZÉQUEL \\
\hline Nomada marshamella & $1 \mathrm{~F}$ & 01/07/2017 & P. JÉZÉQUEL & P. JÉZÉQUEL & É. DUFRÊNE & P. JÉZÉQUEL \\
\hline Nomada ruficornis & $1 \mathrm{~F}$ & 08/04/2017 & P. JÉZÉQUEL & P. JÉZÉQUEL & É. DUFRÊNE & P. JÉZÉQUEL \\
\hline Nomada succincta & $2 \mathrm{~F}$ & 08/04/2017 & P. JÉZÉQUEL & P. JÉZÉQUEL & É. DUFRÊNE & P. JÉZÉQUEL \\
\hline Nomada zonata & $1 \mathrm{~F}$ & $02 / 06 / 2017$ & P. JÉZÉQUEL & P. JÉZÉQUEL & É. DUFRÊNE & P. JÉZÉQUEL \\
\hline \multicolumn{7}{|l|}{ Colletidae } \\
\hline Hylaeus dilatatus & $1 \mathrm{~F}$ & $18 / 06 / 2017$ & P. JÉZÉQUEL & S. GADOUM & & P. JÉZÉQUEL \\
\hline Hylaeus communis & $1 \mathrm{~F}+1 \mathrm{M}$ & $01 / 08 / 2017$ & P. JÉZÉQUEL & P. JÉZÉQUEL & S. GADOUM & P. JÉZÉQUEL \\
\hline Hylaeus hyalinatus & $1 \mathrm{M}$ & $18 / 06 / 2017$ & P. JÉZÉQUEL & P. JÉZÉQUEL & S. GADOUM & P. JÉZÉQUEL \\
\hline Hylaeus variegatus & $1 \mathrm{~F}$ & 01/09/2017 & P. JÉZÉQUEL & P. JÉZÉQUEL & S. GADOUM & P. JÉZÉQUEL \\
\hline \multicolumn{7}{|l|}{ Halictidae } \\
\hline Halictus langobardicus & $4 \mathrm{M}$ & $01 / 08 / 2017$ & P. JÉZÉQUEL & P. JÉZÉQUEL & D. GENOUd & P. JÉZÉQUEL \\
\hline Halictus maculatus & $4 \mathrm{~F}+2 \mathrm{M}$ & $01 / 07 / 2017$ & P. JÉZÉQUEL & P. JÉZÉQUEL & & P. JÉZÉQUEL \\
\hline Halictus scabiosae & $2 \mathrm{~F}+1 \mathrm{M}$ & $05 / 05 / 2017$ & P. JÉZÉQUEL & P. JÉZÉQUEL & & P. JÉZÉQUEL \\
\hline
\end{tabular}




\begin{tabular}{|c|c|c|c|c|c|c|}
\hline Halictus gr. simplex & $5 \mathrm{~F}$ & 05/05/2017 & P. JÉZÉQUEL & P. JÉZÉQUEL & & P. JÉZÉQUEL \\
\hline Halictus subauratus & $3 \mathrm{~F}+2 \mathrm{M}$ & $01 / 07 / 2017$ & P. JÉZÉQUEL & P. JÉZÉQUEL & & P. JÉZÉQUEL \\
\hline Halictus tumulorum & $41 \mathrm{~F}+9 \mathrm{M}$ & $21 / 05 / 2017$ & P. JÉZÉQUEL & P. JÉZÉQUEL & & P. JÉZÉQUEL \\
\hline Lasioglossum calceatum & $5 \mathrm{~F}+2 \mathrm{M}$ & 01/07/2017 & P. JÉZÉQUEL & P. JÉZÉQUEL & & P. JÉZÉQUEL \\
\hline Lasioglossum laticeps & $2 \mathrm{~F}$ & $01 / 08 / 2017$ & P. JÉZÉQUEL & P. JÉZÉQUEL & & P. JÉZÉQUEL \\
\hline Lasioglossum lativentre & $32 \mathrm{~F}+7 \mathrm{M}$ & 01/06/2017 & P. JÉZÉQUEL & P. JÉZÉQUEL & & P. JÉZÉQUEL \\
\hline Lasioglossum leucozonium & $5 \mathrm{~F}$ & 01/07/2017 & P. JÉZÉQUEL & P. JÉZÉQUEL & & P. JÉZÉQUEL \\
\hline Lasioglossum malachurum & $41 \mathrm{~F}+3 \mathrm{M}$ & $01 / 06 / 2017$ & P. JÉZÉQUEL & P. JÉZÉQUEL & & P. JÉZÉQUEL \\
\hline Lasioglossum minutissimum & $1 \mathrm{~F}$ & 01/08/2017 & P. JÉZÉQUEL & P. JÉZÉQUEL & & P. JÉZÉQUEL \\
\hline Lasioglossum morio & $37 \mathrm{~F}+12 \mathrm{M}$ & $22 / 04 / 2017$ & P. JÉZÉQUEL & P. JÉZÉQUEL & & P. JÉZÉQUEL \\
\hline Lasioglossum pauxilum & $1 \mathrm{~F}$ & 01/08/2017 & P. JÉZÉQUEL & P. JÉZÉQUEL & & P. JÉZÉQUEL \\
\hline Lasioglossum sexnotatum & $1 \mathrm{~F}$ & 01/09/2017 & P. JÉZÉQUEL & P. JÉZÉQUEL & & P. JÉZÉQUEL \\
\hline Lasioglossum villosolum & $1 \mathrm{~F}$ & $01 / 08 / 2017$ & P. JÉZÉQUEL & P. JÉZÉQUEL & & P. JÉZÉQUEL \\
\hline Lasioglossum xanthopus & $1 \mathrm{~F}$ & 01/06/2017 & P. JÉZÉQUEL & P. JÉZÉQUEL & & P. JÉZÉQUEL \\
\hline Lasioglossum zonulum & $4 \mathrm{~F}$ & 01/08/2017 & P. JÉZÉQUEL & P. JÉZÉQUEL & & P. JÉZÉQUEL \\
\hline Lasioglossum spp. & 18 & 01/08/2017 & P. JÉZÉQUEL & P. JÉZÉQUEL & & P. JÉZÉQUEL \\
\hline Sphecodes ephippius & $1 \mathrm{~F}+2 \mathrm{M}$ & 01/07/2017 & P. JÉZÉQUEL & P. JÉZÉQUEL & É. DUFRÊNE & P. JÉZÉQUEL \\
\hline Sphecodes gibus & $1 \mathrm{M}$ & $01 / 072017$ & P. JÉZÉQUEL & É. DUFRÊNE & & P. JÉZÉQUEL \\
\hline Sphecodes hyalinatus & $1 \mathrm{~F}$ & $01 / 08 / 2017$ & P. JÉZÉQUEL & É. DUFRÊNE & & P. JÉZÉQUEL \\
\hline Sphecodes molinicornis & $4 \mathrm{~F}+1 \mathrm{M}$ & $01 / 06 / 2017$ & P. JÉZÉQUEL & P. JÉZÉQUEL & É. DUFRÊNE & P. JÉZÉQUEL \\
\hline Sphecodes puncticeps & $3 \mathrm{~F}$ & 01/06/2017 & P. JÉZÉQUEL & P. JÉZÉQUEL & É. DUFRÊNE & P. JÉZÉQUEL \\
\hline Sphecodes reticulatus & $1 \mathrm{M}$ & 01/06/2017 & P. JÉZÉQUEL & P. JÉZÉQUEL & É. DUFRÊNE & P. JÉZÉQUEL \\
\hline \multicolumn{7}{|l|}{ Megachilidae } \\
\hline Anthidium manicatum & $1 \mathrm{M}$ & $01 / 072017$ & P. JÉZÉQUEL & P. JÉZÉQUEL & & P. JÉZÉQUEL \\
\hline Chelostoma distinctum & $1 \mathrm{~F}$ & $01 / 08 / 2017$ & P. JÉZÉQUEL & P. JÉZÉQUEL & M. AUBERT & P. JÉZÉQUEL \\
\hline Heriades truncorum & $1 \mathrm{~F}$ & 01/07/2017 & P. JÉZÉQUEL & P. JÉZÉQUEL & M. AUBERT & P. JÉZÉQUEL \\
\hline Hoplitis leucomelana & $1 \mathrm{~F}+1 \mathrm{M}$ & $01 / 06 / 2017$ & P. JÉZÉQUEL & P. JÉZÉQUEL & M. AUBERT & P. JÉZÉQUEL \\
\hline Hoplitis tridentata & $1 \mathrm{~F}$ & 01/07/2017 & P. JÉZÉQUEL & P. JÉZÉQUEL & M. AUBERT & P. JÉZÉQUEL \\
\hline Megachile centuncularis & $3 \mathrm{~F}+2 \mathrm{M}$ & $01 / 06 / 2017$ & P. JÉZÉQUEL & P. JÉZÉQUEL & M. AUBERT & P. JÉZÉQUEL \\
\hline Megachile lagopoda & $1 \mathrm{M}$ & 01/08/2017 & P. JÉZÉQUEL & P. JÉZÉQUEL & M. AUBERT & P. JÉZÉQUEL \\
\hline Osmia caerulescens & $2 \mathrm{M}$ & $22 / 04 / 2017$ & P. JÉZÉQUEL & P. JÉZÉQUEL & M. AUBERT & P. JÉZÉQUEL \\
\hline Osmia cornuta & $1 \mathrm{~F}$ & 08/04/2017 & P. JÉZÉQUEL & P. JÉZÉQUEL & & P. JÉZÉQUEL \\
\hline Osmia bicornis & $1 \mathrm{~F}$ & 05/05/2017 & P. JÉZÉQUEL & P. JÉZÉQUEL & & P. JÉZÉQUEL \\
\hline Osmia gallarum & $2 \mathrm{~F}$ & $21 / 05 / 2017$ & P. JÉZÉQUEL & P. JÉZÉQUEL & M. AUBERT & P. JÉZÉQUEL \\
\hline Stelis punctulatissima & $1 \mathrm{M}$ & 01/07/2017 & P. JÉZÉQUEL & P. JÉZÉQUEL & É. DUFRÊNE & P. JÉZÉQUEL \\
\hline \multicolumn{7}{|l|}{ Melittidae } \\
\hline Melitta leporina & $3 \mathrm{~F}$ & 01/07/2017 & P. JÉZÉQUEL & D. GENOUD & & P. JÉZÉQUEL \\
\hline
\end{tabular}

70 espèces

423 individus

Andrenidae

$\begin{array}{ll}\text { Andrena alfkenella } & 1 \mathrm{~F} \\ \text { Andrena cineraria } & 6 \mathrm{~F} \\ \text { Andrena haemorroha } & 1 \mathrm{~F}+1 \mathrm{M} \\ \text { Andrena labiata } & 1 \mathrm{~F} \\ \text { Andrena nigroaena } & 1 \mathrm{~F}\end{array}$

\section{Récoltes 2018 (provisoire)}

$\begin{array}{lllll}\text { 12/05/2018 } & \text { P. JÉZÉQUEL } & \text { P. JÉZÉQUEL } & \text { à confirmer } & \text { P. JÉZÉQUEL } \\ 22 / 04 / 2018 & \text { P. JÉZÉQUEL } & \text { P. JÉZÉQUEL } & \text { P. JÉZÉQUEL } \\ \text { 22/04/2018 } & \text { P. JÉZÉQUEL } & \text { P. JÉZÉQUEL } & \text { P. JÉZÉQUEL } \\ \text { 12/05/2018 } & \text { P. JÉZÉQUEL } & \text { P. JÉZÉQUEL } & \text { P. JÉZÉQUEL } \\ \text { 22/04/2018 } & \text { P. JÉZÉQUEL } & \text { P. JÉZÉQUEL } & \text { P. JÉZÉQUEL }\end{array}$




\begin{tabular}{|c|c|c|c|c|c|c|}
\hline Andrena nidida & $1 \mathrm{~F}$ & $12 / 05 / 2018$ & P. JÉZÉQUEL & P. JÉZÉQUEL & & P. JÉZÉQUEL \\
\hline \multicolumn{7}{|l|}{ Apidae } \\
\hline Anthophora retusa & $1 \mathrm{~F}$ & $30 / 04 / 2018$ & P. JÉZÉQUEL & P. JÉZÉQUEL & P. RASMONT & P. JÉZÉQUEL \\
\hline Bombus lapidarius & $1 \mathrm{~F}$ & $18 / 03 / 2018$ & P. JÉZÉQUEL & P. JÉZÉQUEL & & P. JÉZÉQUEL \\
\hline Tetraloniella alticincta & $1 \mathrm{~F}$ & $22 / 07 / 2018$ & P. JÉZÉQUEL & P. JÉZÉQUEL & M. AUBERT & P. JÉZÉQUEL \\
\hline Xylocopa violacea & $1 \mathrm{~F}$ & $14 / 04 / 2018$ & P. JÉZÉQUEL & P. JÉZÉQUEL & & P. JÉZÉQUEL \\
\hline \multicolumn{7}{|l|}{ Megachilidae } \\
\hline Anthidium oblongatum & $1 \mathrm{~F}$ & $22 / 07 / 2018$ & P. JÉZÉQUEL & P. JÉZÉQUEL & M. AUBERT & P. JÉZÉQUEL \\
\hline Chelostoma florissomne & $1 \mathrm{~F}$ & $12 / 05 / 2018$ & P. JÉZÉQUEL & P. JÉZÉQUEL & M. AUBERT & P. JÉZÉQUEL \\
\hline Megachile ericetorum & $1 \mathrm{~F}$ & $22 / 07 / 2018$ & P. JÉZÉQUEL & P. JÉZÉQUEL & M. AUBERT & P. JÉZÉQUEL \\
\hline Stelis breviuscula & $1 \mathrm{M}$ & $22 / 07 / 2018$ & P. JÉZÉQUEL & P. JÉZÉQUEL & M. AUBERT & P. JÉZÉQUEL \\
\hline
\end{tabular}

14 espèces

20 individus

84 espèces au total

Les 70 espèces observées en 2017 dans cette serre représentent $34 \%$ du cortège d'espèces recensées à l'échelle du département d'Ille-et-Vilaine (35). Cette méthode permet donc d'obtenir des résultats significatifs tant à l'échelle locale (dans le cadre d'un $\mathrm{ABC}$ communal par exemple) que régionale.

Quatre des espèces capturées dans la serre sont nouvelles pour la région Bretagne, telle qu'Hoplitis tridendata signalée uniquement dans les départements 49 (Maine-et-Loire) et 50 (Manche) au sein de la liste des abeilles du Massif armoricain (regroupant 12 départements ${ }^{1}$ appartenant aux régions Bretagne, Normandie et Pays de la Loire).

On peut remarquer que certaines espèces communes à Rennes sont absentes de l'inventaire, telles qu'Anthophora plumipes que l'on trouve beaucoup en milieu semi urbain et jusqu'au centre-ville, Andrena cineraria qui sera finalement collectée en 2018 ou encore Bombus lapidarius observé dans la parcelle en 2018.

Pour 2018, les premiers résultats donnent 14 nouvelles espèces non recensées l'année passée (tableau II). Parmi ces 14 espèces, deux sont remarquables car nouvelles à l'échelle du département (Tetraloniella alticincta) ou de la région Bretagne (Stelis breviuscula).

\footnotetext{
${ }^{1}$ Départements du Massif armoricain :

- en Normandie : Calvados (14), Manche (50) et Orne (61)

- en Bretagne : Côtes-d'Armor (22), Finistère (29), Ille-et-Vilaine (35) et Morbihan (56).

- en Pays de la Loire: Loire-Atlantique (44), Maine-et-Loire (49), Mayenne (53), Sarthe (72) et Vendée (85)

Note : les Deux-Sèvres (79) en Nouvelle-Aquitaine comprennent aussi une partie du Massif armoricain au sens géologique.
}

Il y a donc un intérêt qualitatif et quantitatif à la méthode de collecte par les serres. En outre, les jardins potagers sont présents sur la grande majorité du territoire et dans tout type de milieu, y compris urbain, ce qui permet aisément l'obtention de données dans une grande diversité de paysages et d'environnements.

\section{Comparaison avec les autres serres}

Les résultats des collectes dans les serres prospectées à Rennes en août 2017 sont très variables, allant de 90 à 200 Abeilles. C'est beaucoup moins que la serre du type de la figure 1 (Perma G), ceci pour de multiples raisons telles que la difficulté d'extraction, la taille des ouvertures et les habitudes du jardinier. Les pratiques $\mathrm{du}$ jardinier sont particulièrement importantes : certains réalisent des semis dès le mois de mars-avril alors que d'autres n'utilisent leur serre qu'à partir de mai-juin.

Certaines personnes, comme des retraités par exemple, visitent régulièrement leurs serres et les portes restent fréquemment ouvertes, alors que d'autres les laissent fermées toute la semaine jusqu'aux grosses chaleurs de juin et ne viennent qu'un après-midi par semaine.

Pour témoigner de la faible collecte de certaines serres, on peut prendre l'exemple de la serre de la figure 4 (Gayeulles-est numéro 1 à Rennes) qui, en 2017, n'a collecté que 90 Abeilles (tableau III). Elle est pourtant du même type que la serre de la figure 1 (423 Abeilles) dans laquelle les abeilles 
sont très bien collectées et faciles à récolter. Elle est par ailleurs située dans un jardin ouvrier avec une vingtaine de parcelles de $50 \mathrm{~m}^{2}$ autour, près d'un boisement et d'une zone humide.

Tableau III. Collectes de la serre Gayeulles-est numéro 1 (Gayeulles) : bilan.

\begin{tabular}{lcc} 
Famille & Nombre & Proportion \\
\hline Halictidae & 45 & $50 \%$ \\
Megachilidae & 16 & $17,80 \%$ \\
Andrenidae & 14 & $15,50 \%$ \\
Apidae & 4 & $4,50 \%$ \\
Colletidae & 3 & $3,30 \%$ \\
Mellitidae & 8 & $8,90 \%$ \\
Total & 90 & $100 \%$
\end{tabular}

Plusieurs hypothèses peuvent être avancées pour expliquer la faible collecte constatée :

- Dilution des captures dans les autres serres qui, elles, ne collectent pas ;

- Absence de linéaire principal dans ce dédale de jardins ;

- Présence d'une seule porte (contre 2 pour la serre de la figure 1), celle-ci n'étant qu'à moitié ouverte la plupart du temps ;

- Saison du jardinier initiée seulement au mois de mai ;

- Fermeture fréquente de la serre pendant la semaine.

Aussi, les opportunités de captures sont moins nombreuses et essentiellement estivales comme le confirme l'inventaire (tableau IV).

Avec 14 individus, les Andrenidae ne représentent que $15 \%$ du nombre total des abeilles capturées, avec six espèces tardives ou à deux générations : Andrena bicolor, A. dorsata, A. flavipes, A. fulvago, A. minitula, A. ovatula. Ils représentaient $22 \%$ pour la serre Perma $G$ à la Taupinais.

Les Megachilidae avec 16 spécimens représentent $18 \%$ du nombre total des abeilles capturées avec 9 espèces contre $4 \%$ dans la serre Perma G. Les espèces collectées sont plutôt intéressantes : Anthidium mancatum, Anthidelium strigatum**, Heriades truncurum, Hoplitis leucolamena**, Megachile centuncularis, $M$. versicolor, $M$. rotundata*, Osmia cornuta, Pseudoanthidium nanum $^{* *}$, Stelis breviuscula**, et S. ornatula**. Cette dernière n'était répertoriée que dans un seul autre département (Calvados) du Massif armoricain. Concernant Pseudoanthidium nanum, il s'agit de la première et seule donnée pour l'ensemble du Massif armoricain qui comprend pourtant 12 départements.

La connaissance des usages et habitudes du jardinier est donc à prendre en considération lors de la préparation d'un inventaire par cette méthode d'échantillonnage au même titre que les autres facteurs (paysage, cultures ...) ; on constate ainsi que, dans cet exemple, les Abeilles printanières sont sous-représentées. Les grandes comme les petites collectes donnent des résultats avec de nouvelles espèces pour la Bretagne dont certaines rares dans le Massif Armoricain.

\section{Conclusion}

La diversité des serres (taille, structure, usage, localisation) rend difficile la mise en place d'un protocole standardisé et l'obtention de données facilement comparables à des fins scientifiques. Cependant, le renouvellement des inventaires sur plusieurs années, et la multiplicité des serres à l'échelle du territoire national, réparties dans une grande diversité d'habitats et d'environnements, permettrait de gommer la forte variabilité locale due à la spécificité de chaque serre.

L'intérêt majeur de cette méthode de collecte est avant tout la possibilité de réaliser un inventaire à large échelle et de compléter les listes d'abeilles locales et régionales. La mise en œuvre est simple grâce à une collecte de proximité qui peut être réalisée par des non experts. L'aspect passif de cette méthode (pas de capture au filet, pas de pose de pièges) devrait permettre de faciliter son acceptabilité auprès du grand public et des nombreux naturalistes refusant le piégeage actif, soucieux de leur potentiel impact sur les communautés de pollinisateurs.

C'est également l'occasion de développer un réseau dynamique, pouvant produire des données tant à l'échelle locale que régionale, et pouvant intéresser les réseaux naturalistes associatifs comme scientifiques, les collectivités territoriales et services de l'État tels que les DREAL (notamment dans le cadre du PNA pollinisateurs). Une campagne d'inventaires même régionale nécessiterait des moyens pour la mise en collection et l'identification de ces montagnes d'Abeilles qui, on l'espère, ne seraient alors plus perdues pour la science. 
Tableau IV. Collectes de la serre Gayeulles-est numéro 1 (Rennes) : détail.

Coordonnées Lambert $93:$ X $355018-$ Y 6791686. $\mathrm{F}=$ femelle. $\mathrm{M}=$ mâle.

Toutes les collectes sont ici de 2017.

\begin{tabular}{|c|c|c|c|c|c|c|}
\hline Espèce & Effectif & Date & Récolteur & Déterminateur 1 & Déterminateur 2 & Collection \\
\hline \multicolumn{7}{|l|}{ Andrenidae } \\
\hline Andrena bicolor & $3 \mathrm{~F}$ & $15 / 08 / 2017$ & P. JÉZÉQUEL & P. JÉZÉQUEL & D. GENOUD & P. JÉZÉQUEL \\
\hline Andrena dorsata & $2 \mathrm{~F}$ & $15 / 08 / 2017$ & P. JÉZÉQUEL & P. JÉZÉQUEL & D. GENOUD & P. JÉZÉQUEL \\
\hline Andrena lavipes & $1 \mathrm{~F}$ & $15 / 08 / 2017$ & P. JÉZÉQUEL & P. JÉZÉQUEL & D. GENOUD & P. JÉZÉQUEL \\
\hline Andrena fulvago & $2 \mathrm{~F}$ & $15 / 08 / 2017$ & P. JÉZÉQUEL & P. JÉZÉQUEL & D. GENOUD & P. JÉZÉQUEL \\
\hline Andrena minitula & $1 \mathrm{~F}$ & $15 / 08 / 2017$ & P. JÉZÉQUEL & P. JÉZÉQUEL & D. GENOUD & P. JÉZÉQUEL \\
\hline Andrena (Micrandrena) spp. & $4 \mathrm{~F}$ & $15 / 08 / 2017$ & P. JÉZÉQUEL & P. JÉZÉQUEL & & P. JÉZÉQUEL \\
\hline Andrena ovatula & $1 \mathrm{~F}$ & $15 / 08 / 2017$ & P. JÉZÉQUEL & D. GENOUD & & P. JÉZÉQUEL \\
\hline \multicolumn{7}{|l|}{ Apidae } \\
\hline Apis mellifera & $\mathrm{NC}$ & $15 / 08 / 2017$ & P. JÉZÉQUEL & P. JÉZÉQUEL & & P. JÉZÉQUEL \\
\hline Anthophora plumipes & $1 \mathrm{M}$ & $15 / 08 / 2017$ & P. JÉZÉQUEL & P. JÉZÉQUEL & & P. JÉZÉQUEL \\
\hline Bombus pascuorum & $1 \mathrm{~F}$ & $15 / 08 / 2017$ & P. JÉZÉQUEL & G. MAHÉ & & P. JÉZÉQUEL \\
\hline Ceratina cyanea & $1 \mathrm{~F}$ & $15 / 08 / 2017$ & P. JÉZÉQUEL & P. JÉZÉQUEL & & P. JÉZÉQUEL \\
\hline Nomada marshamella & $1 \mathrm{~F}$ & $15 / 08 / 2017$ & P. JÉZÉQUEL & P. JÉZÉQUEL & É. DUFRÊNE & P. JÉZÉQUEL \\
\hline \multicolumn{7}{|l|}{ Colletidae } \\
\hline Hylaeus hyalinatus & $1 \mathrm{~F}$ & $15 / 08 / 2017$ & P. JÉZÉQUEL & P. JÉZÉQUEL & S. GADOUM & P. JÉZÉQUEL \\
\hline Hylaeus punctulatissimus & $1 \mathrm{~F}+1 \mathrm{M}$ & $15 / 08 / 2017$ & P. JÉZÉQUEL & S. GADOUM & & P. JÉZÉQUEL \\
\hline \multicolumn{7}{|l|}{ Halictidae } \\
\hline Halictus langobardicus & $4 \mathrm{M}$ & $15 / 08 / 2017$ & P. JÉZÉQUEL & P. JÉZÉQUEL & D. GENOUD & P. JÉZÉQUEL \\
\hline Halictus scabiosae & $1 \mathrm{~F}$ & $15 / 08 / 2017$ & P. JÉZÉQUEL & P. JÉZÉQUEL & & P. JÉZÉQUEL \\
\hline Halictus gr. simplex & $3 \mathrm{~F}$ & $15 / 08 / 2017$ & P. JÉZÉQUEL & P. JÉZÉQUEL & & P. JÉZÉQUEL \\
\hline Halictus tumulorum & $5 \mathrm{~F}+2 \mathrm{M}$ & $15 / 08 / 2017$ & P. JÉZÉQUEL & P. JÉZÉQUEL & & P. JÉZÉQUEL \\
\hline Lasioglossum albipes & $1 \mathrm{~F}$ & $15 / 08 / 2017$ & P. JÉZÉQUEL & P. JÉZÉQUEL & à confirmer & P. JÉZÉQUEL \\
\hline Lasioglossum laticeps & $2 \mathrm{~F}+1 \mathrm{M}$ & $15 / 08 / 2017$ & P. JÉZÉQUEL & P. JÉZÉQUEL & & P. JÉZÉQUEL \\
\hline Lasioglossum malachurum & $11 \mathrm{~F}$ & $15 / 08 / 2017$ & P. JÉZÉQUEL & P. JÉZÉQUEL & & P. JÉZÉQUEL \\
\hline Lasioglossum morio & $7 \mathrm{~F}$ & $15 / 08 / 2017$ & P. JÉZÉQUEL & P. JÉZÉQUEL & & P. JÉZÉQUEL \\
\hline Lasioglossum spp. & 6 ind. & $15 / 08 / 2017$ & P. JÉZÉQUEL & P. JÉZÉQUEL & & P. JÉZÉQUEL \\
\hline Sphecodes ephippius & $1 \mathrm{M}$ & $15 / 08 / 2017$ & P. JÉZÉQUEL & P. JÉZÉQUEL & É. DUFRÊNE & P. JÉZÉQUEL \\
\hline Sphecodes molinicornis & $1 \mathrm{M}$ & $15 / 08 / 2017$ & P. JÉZÉQUEL & É. DUFRÊNE & & P. JÉZÉQUEL \\
\hline \multicolumn{7}{|l|}{ Megachilidae } \\
\hline Anthidium manicatum & $1 \mathrm{~F}$ & $15 / 08 / 2017$ & P. JÉZÉQUEL & P. JÉZÉQUEL & & P. JÉZÉQUEL \\
\hline Anthidiellum strigatum & $1 \mathrm{~F}$ & $15 / 08 / 2017$ & P. JÉZÉQUEL & P. JÉZÉQUEL & M. AUBERT & P. JÉZÉQUEL \\
\hline Heriades truncorum & $2 \mathrm{~F}$ & $15 / 08 / 2017$ & P. JÉZÉQUEL & P. JÉZÉQUEL & M. AUBERT & P. JÉZÉQUEL \\
\hline Hoplitis leucomelana & $1 \mathrm{~F}$ & $15 / 08 / 2017$ & P. JÉZÉQUEL & P. JÉZÉQUEL & M. AUBERT & P. JÉZÉQUEL \\
\hline Megachile centuncularis & $3 \mathrm{~F}$ & $15 / 08 / 2017$ & P. JÉZÉQUEL & P. JÉZÉQUEL & M. AUBERT & P. JÉZÉQUEL \\
\hline Megachile rotundata & $1 \mathrm{~F}$ & $15 / 08 / 2017$ & P. JÉZÉQUEL & P. JÉZÉQUEL & M. AUBERT & P. JÉZÉQUEL \\
\hline Osmia cornuta & $1 \mathrm{~F}$ & $15 / 08 / 2017$ & P. JÉZÉQUEL & P. JÉZÉQUEL & & P. JÉZÉQUEL \\
\hline Pseudoanthidium nanum & $1 \mathrm{~F}$ & $15 / 08 / 2017$ & P. JÉZÉQUEL & P. JÉZÉQUEL & M. AUBERT & P. JÉZÉQUEL \\
\hline Stelis breviuscula & $3 \mathrm{M}$ & $15 / 08 / 2017$ & P. JÉZÉQUEL & P. JÉZÉQUEL & É. DUFRÊNE & P. JÉZÉQUEL \\
\hline Stelis ornatula & $2 \mathrm{~F}$ & $15 / 08 / 2017$ & P. JÉZÉQUEL & P. JÉZÉQUEL & É. DUFRÊNE & P. JÉZÉQUEL \\
\hline \multicolumn{7}{|l|}{ Melittidae } \\
\hline Macropis europea & $8 \mathrm{~F}$ & $15 / 08 / 2017$ & P. JÉZÉQUEL & P. JÉZÉQUEL & & P. JÉZÉQUEL \\
\hline 33 espèces & 90 individus & & & & & \\
\hline
\end{tabular}

D’un autre côté, ces captures répétées chaque année peuvent potentiellement, en cas de captures importantes, affecter les communautés d'insectes présents autour des serres. L'augmentation de la pratique des jardins partagés et le développement des maraîchers devraient inciter les fabricants de 
serres à aménager de petites ouvertures renforcées aux angles qu'il serait possible de fermer avec des bouchons lors d'épisodes venteux pour éviter les effets de pressions et dépressions qui peuvent arracher la serre de son assise au sol ou déchirer la bâche. Une étude sur les tunnels des grandes exploitations agricoles et pépinières devrait pouvoir évaluer les impacts des serres, en termes de dynamique des populations, sur l'entomofaune.

\section{Remerciements}

Je remercie Mickaël HARDY, maraîcher à Perma $G$, et tous les jardiniers qui m'ont donné accès à leurs serres. J'exprime ma gratitude à David GEnoud, Éric DufrÊnE, Gille MAHÉ, Mathieu AUBERT et Serge GADOUM pour les déterminations des Abeilles ou leur confirmation. Je remercie également Benoît GESLIN et Tanguy JEAN pour leurs conseils. Enfin, j’adresse mes remerciements à Maël GARIN et aux bénévoles des associations Bretagne Vivante et GRÉTIA.

\section{Bibliographie}

Amiet F, 1996. Insecta helvetica 12. Fauna Hymenoptera, Apidae, 1. Teil. Schweizerischen Entomologischen Gesellschaft, Zürich (Confédération helvétique), et Centre Suisse de Cartographie de la Faune, Neûchatel (Confédération helvétique), 98 pp.

Amiet F, MÜller A \& Neumeyer R, 1999. Fauna helvetica 4. Apidae 2. Schweizerischen Entomologischen Gesellschaft, Zürich (Confédération helvétique), et Centre Suisse de Cartographie de la Faune, Neûchatel (Confédération helvétique), 219 pp.

Amiet F, Herrmann M, Müller A \& NeUmeyer R, 2001. Fauna helvetica 6. Apidae 3. Schweizerischen Entomologischen Gesellschaft, Zürich (Confédération helvétique), et Centre Suisse de Cartographie de la Faune, Neûchatel (Confédération helvétique), 208 pp.

Amiet F, Herrmann M, Müller A \& NeUmeyer R, 2004. Fauna helvetica 9. Apidae 4. Schweizerischen Entomologischen Gesellschaft, Zürich (Confédération helvétique), et Centre Suisse de Cartographie de la Faune, Neûchatel (Confédération helvétique), 273 pp.
Amiet F, HerrmanN M, Müller A \& NeUmeYer R, 2007. Fauna helvetica 20. Apidae 5. Schweizerischen Entomologischen Gesellschaft, Zürich (Confédération helvétique), et Centre Suisse de Cartographie de la Faune, Neûchatel (Confédération helvétique), 356 pp.

Amiet F, Herrmann M, Müller A \& NeUmeYer R, 2010. Fauna helvetica 26. Apidae 6. Schweizerischen Entomologischen Gesellschaft, Zürich (Confédération helvétique), et Centre Suisse de Cartographie de la Faune, Neûchatel (Confédération helvétique), $317 \mathrm{pp}$.

Amiet F, Müller A \& Neumeyer R, 2014. Fauna helvetica 4. Apidae 2. $2^{\mathrm{e}}$ édition revue et corrigée (1e édition 1999). Schweizerischen Entomologischen Gesellschaft, Zürich (Confédération helvétique), et Centre Suisse de Cartographie de la Faune, Neûchatel (Confédération helvétique), 239 pp.

Amiet F, MÜller \& Praz C, 2017. Fauna helvetica 29. Apidae 1. $2^{\mathrm{e}}$ édition revue et corrigée (1 ${ }^{\mathrm{e}}$ édition 1996). Schweizerischen Entomologischen Gesellschaft, Zürich (Confédération helvétique), et Centre Suisse de Cartographie de la Faune, Neûchatel (Confédération helvétique), $187 \mathrm{pp}$.

APOIDEA ARMORICANA, 2018. Listes départementales des abeilles de Bretagne, Pays-dela-Loire et Basse Normandie. Observatoire des Abeilles, Flines-lez-Raches (France).

https://oabeilles.net/les-abeilles-de-france (consulté en 2019)

Patiny S \& Terzo M, 2010. Catalogue et clé des sous-genres et espèces du genre Andrena de Belgique et du nord de la France. Laboratoire de Zoologie, Université de Mons (Belgique), 39 pp.

www.atlashymenoptera.net/biblio/Patiny \& Terz o_2010_Andrena_Belgique_et_N_France_compact .pdf (consulté en 2019)

Pauly A, 2015. Clés illustrées pour l'identification des abeilles de Belgique et des régions limitrophes (hymenoptera apoida). I. Halictidae. Document de Travail du projet BELBEES, 14 novembre 2015, $118 \mathrm{pp}$.

http://www.atlashymenoptera.net/biblio/Pauly_20 $15 \mathrm{Cl} \% \mathrm{C} 3 \% \mathrm{~A} 9 \mathrm{~s}$ Halictidae Belgique.pdf

(consulté en 2019) 
Pauly A, 2015. Clés illustrées pour l'identification des abeilles de Belgique. II. Megachilidae. Document de Travail du projet BELBEES, $61 \mathrm{pp}$. http://zoologie.umons.ac.be/hymenoptera/biblio/P auly_2015_Cl\%C3\%A9s_Megachilidae_Belgique 9JAN2016.pdf (consulté en 2019)

Pauly A, 2016. Les espèces du genre Sphecodes Latreille, 1804, en Belgique. Document de travail du projet BELBEES, $93 \mathrm{pp}$.

http://zoologie.umons.ac.be/hymenoptera/biblio/P auly_2016_Sphecodes\%20Belgique\%20LQ.pdf (consulté en 2019) 